\title{
Neopterin and soluble CD14 levels as indicators of immune activation in cases with low anti-HCV reactivity and true $\mathrm{HCV}$ infection
}

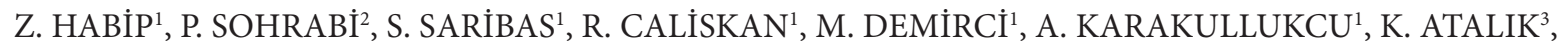 \\ P. YUKSEL ${ }^{1}$, O. UYSAL ${ }^{4}$, E. KOSAN ${ }^{5}$, H. BAHAR TOKMAN ${ }^{1}$, B. KOCAZEYBEK ${ }^{*}$
}

\begin{abstract}
${ }^{1}$ Cerrahpasa Faculty of Medicine, Department of Medical Microbiology, Istanbul University, Cerrahpasa Street, 34098, Istanbul, Turkey; ${ }^{2}$ Istanbul Public Health Laboratory, Minister of Health of the Republic of Turkey, Istanbul, Turkey; ${ }^{3}$ Department of Medical Microbiology, Istanbul Medical Faculty Istanbul University, Istanbul, Turkey; ${ }^{4}$ Deparment of Biostatistics, Medical School of Bezmialem Vakif University, Istanbul, Turkey; ${ }^{5}$ Turkish Red Crescent, North Marmara Blood Center, Istanbul, Turkey
\end{abstract}

\begin{abstract}
Summary. - Neopterin and soluble CD14 (sCD14) are detected at high levels in hepatitis C virus (HCV) infections. We aimed to evaluate the role of these plasma immune activation biomarkers, for the indirect assessment of immune activation status of patients with low anti-HCV reactivity and a HCV infection. Low anti-HCV reactivity group (LRG, n: 70), true positive HCV infection group (THG, 30) and healthy control group (HCG, 30) were analyzed in this study. We have used ELISA, HCV RIBA/LIA and HCV-RNA methods. Mean neopterin levels were significantly lower in LRG than THG $(\mathrm{p}<0.001)$. In contrast, those values were not significantly different from those of HCG ( $p>0.05)$. Mean $s C D 14$ were significantly higher in LRG than THG and HCG (p <0.05, p <0.001). Values of $3.95 \mu \mathrm{g} / \mathrm{ml}$ and $5.36 \mathrm{nmol} / \mathrm{l}$ for sCD14 and neopterin resulted in the maximum area under the receiver operating characteristic curves (ROC), which were 0.859 (95\% CI, 0.745 to $0.935 ;<0.0001)$ and 0.788 (95\% CI, 0.663 to 0.883 ; <0.0001), respectively. These cut-offs corresponded to a sensitivity of $73.3 \%$ and a specificity of $73.3 \%$ for neopterin and of $100 \%$ and $76.7 \%$ for sCD14. Our results suggest that a specific immunoactivation might be caused by true positive HCV infection. Due to the significant results SCD14 in LRG might be non-specifically affected by some underlying atypical immunohematological pathologies. Only neopterin might be used to exclude low anti-HCV reactivity from a true HCV infection. The use of neopterin but not sCD14 in combination with fourth-generation EIA/CMIA combo tests will be useful when nucleic acid tests are not available for screening blood donors at blood banks.
\end{abstract}

Keywords: sCD14; neopterin; HCV infection

\section{Introduction}

Neopterin, which is a pyrazinopyrimidine derivative whose levels increase in pathologies, especially pathologies with activated cellular immune mechanisms, is re-

“Corresponding author. E-mail:bzeybek@istanbul.edu.tr; phone:+90-212-414-30-00/22417, mobile:+90-5076641782.

Abbreviations: $\mathrm{HCG}=$ healthy control group; $\mathrm{HCV}=$ hepatitis $\mathrm{C}$ virus; $\mathrm{LIA}=$ line immunoblot assay; $\mathrm{LRG}=$ low anti-HCV reactivity group; RIBA = recombinant immunoblot assay; $\mathrm{ROC}=$ receiver operating characteristic curves; $\mathrm{sCD} 14=$ soluble CD14; s/co = signal $/$ cut-off values; $\mathrm{THG}=$ true positive $\mathrm{HCV}$ infection group leased from monocytes, macrophages, dendritic cells and endothelial cells activated by interferon gamma (IFN- $\gamma$ ) secreted by Th1 lymphocytes (Hoffmann et al., 2004; Schroecksnadel et al., 2004). Because increased neopterin levels could be an important indicator of a Th1 cellular immune response, this test acquired great clinical and diagnostic importance for atypical HCV laboratory data analysis (Fuchs et al., 1992; Murr et al., 2002). On the other hand, sCD14 is a glycosyl phosphatidyl inositose (GPI) molecule that is expressed on the surface of cells involved in natural immune responses, such as monocyte/macrophages (M/Ms), polymorphonuclear leukocytes (PNLs) and dendritic cells. It has been reported that sCD14 levels could 
be detected at high levels in pathologies, such as trauma, sepsis and rheumatoid arthritis, and in patients with HBV, HCV and HIV infections (Bas et al., 2004; Medhat et al., 2015; Lien et al., 1998). sCD14 could be a specific receptor for the enveloped glycoproteins of $\mathrm{HCV}$, and there appears to be an correlation between the severity of chronic HCV infection and sCD14 levels (Jirillo et al., 1998; Oesterreicher et al., 1995).

Anti-HCV screening tests must be developed continuously due to their low sensitivity and specificity and their frequent use in laboratories. Another shortcoming of these tests is their high level of false positive results in populations with a low level of HCV prevalence $(<3 \%)$. In recent years, fourth-generation EIA and chemiluminescent microparticle immunoassay (CMIA) tests that also target the HCV core antigen with anti-HCV antibodies have been utilized for screening purposes. Recombinant immunoblot assay (RIBA), line immunoblot assay (LIA) and PCR methods have also been used to confirm samples with reactive ELISA test results (Alter et al., 2003; Moyer et al., 1999; Oethinger et al., 2005; Caruntu and Benea, 2006). However, the use of proteins similar to those used in screening tests and the low sensitivity of the tests in comparison to new-generation ELISA tests have made the confirmatory characteristics of RIBA/LIA tests for HCV infections questionable (Pawlotsky et al., 1998; Lok and Gunaratnam, 1997; Dufour et al., 2000).

In recent years, studies associated with the neopterin test, which is used as an additional test in the nonspecific laboratory diagnosis of HCV infection, have attracted considerable attention (Fuchs et al., 1992; Hoffmann et al., 2003).

It was previously suggested that the gastrointestinal system may be a reservoir for the HCV and a possible source of re-infection for hepatocytes through portal vein blood (Miglioresi et al., 2003). Translocation of microbial products into peripheral circulation may cause the loss of gut-associated lymphoid tissue (GALT) integrity. Translocation of microbial products may result in chronic immune activation during both HIV and HCV infections. On the other hand, different mechanisms have been proposed in HIV and HCV infections (abundant proinflammatory cytokine production resulting in gastrointestinal inflammation, dysregulation of T cell subsets, and decreased microbial clearance) (Shata et al., 2013).

In this study, we aimed to evaluate the roles of the plasma immune activation biomarkers neopterin and soluble sCD14 in the indirect assessment of the immune activation status of patients with low anti-HCV reactivity and a true $\mathrm{HCV}$ infection. Besides that, we also aimed to evaluate the neopterin and sCD14 markers for use as surrogate tests in patients with low anti-HCV reactivity, for which molecular methods are not available.

\section{Material and Methods}

Study area and groups. This study was planned as a cross-sectional study and conducted in a case-control-based, descriptive, methodological, and multicenter manner between May 2013 and November 2014 at the following centers: (A) Istanbul University, Cerrahpasa Medical Faculty (IU CTF) Serology/ELISA Laboratory and (B) Turkish Red Crescent Blood Center Laboratory. A total of 70 individuals who were admitted to the facilities for the purpose of clinical diagnosis or blood transfusion constituted low anti-HCV reactivity group, and other groups, which each consisted of 30 people, including a group with true HCV infection and a healthy control group (HCG). The participants in the study were distributed as follows:

1) Low anti-HCV reactivity group (LRG, n: 70): (24 women, 46 men; age range $18-78$ years, average $36.0 \pm 13.2$ years).

2) True positive HCV infection group (THG, n: 30 ): (10 women, 20 men; age range $19-56$ years, average $36.7 \pm 9.2$ years).

3) Healthy control group (HCG, n: 30): (11 women, 19 men; age range 20-57 years, average $35.1 \pm 9.7$ years).

THG was composed of 30 people with chronic HCV who were not undergoing treatment. The patients in this group had reactive anti-HCV test results that were confirmed with the RIBA and/or HCV-RNA tests, and their anti-HIV and HbsAg tests were nonreactive. The HCG was composed of 30 people whose anti-HIV, $\mathrm{HbsAg}$ and anti-HCV tests were non-reactive and who had no viral infections in the past month.

Collection of samples. Ten-milliliter blood samples without anti-coagulant were collected to determine HCV-RNA, neopterin and sCD14 levels. The collected blood samples were centrifuged at 5,000 rpm, and the separated serum and plasma samples were kept at $-80^{\circ} \mathrm{C}$.

Immunological (serologic) methods. At center A, the indicated commercial kits (Nanbase C-96, General Biologicals, Taiwan) were studied using TRITURUS (Diagnostics Grifols, Spain), which is an open and automated system that uses the microelisa method. Signal/cut-off (s/co) values between 3.8 and 1 were evaluated as lowreactive. The Architect anti-HCV (Abbott, USA) kit was studied using the Architect i1000SR (Abbott Laboratories,USA) in a fully automated closed macroelisa system, and s/co values between 1 and 5 were regarded as low-reactive. At center B, the indicated commercial kits (Monolisa anti-HCV PLUS version 2, Bio-Rad, France) were studied using a Freedom Evolyzer 200 (Tecan, Sweden), which is a fully automated ELISA system for the detection of anti-HCV antibodies using the microelisa method. S/co values between 3.8 and 1 were evaluated as low-reactive. Another kit (Liaison L Murex HCV Ab, DiaSorin S.p.A., Italy) was used in a fully automated closed macroelisa system (LiaisonXL, DiaSorin S.p.A., Italy), and s/co values between 1 and 3.8 were considered to be low-reactive. The results obtained from both centers were evaluated according to the recommendations of the employed commercial kits.

HCV RIBA/LIA test. The INNO-LIA ${ }^{\mathrm{ma}}$ HCV Score (Innogenetics, Belgium) is a third-generation LIA that incorporates HCV antigens 
derived from the core region, the E2 hypervariable region (HVR), the NS3 helicase region, and the NS4A, NS4B, and NS5A regions. The LIA method allows for the qualitative determination of anti$\mathrm{HCV}$ antibodies according to every kind of antigen that reacts to human serum. On the test strip, the antigens were coated as 6 discrete lines on a nylon strip with plastic backing. In addition, 4 control lines are coated onto each strip. A test sample is incubated together with the test strip. If HCV antibodies are present in the sample, they will bind to the HCV antigen lines on the strip. Subsequently, an affinity-purified alkaline phosphatase-labeled goat antihuman $\operatorname{IgG}(\mathrm{H}+\mathrm{L})$ conjugate is added and reacts with previously formed specific $\mathrm{HCV}$ antigen/antibody complexes. Incubation with the enzyme substrate produces a chestnut-like color, the intensity of which is proportional to the amount of HCV-specific antibody captured from the sample on any given line. A sample is negative for HCV antibodies if all HCV antigen lines have a negative reactivity rating and if only one $\mathrm{HCV}$ antigen line has a reactivity of \pm , except when reactivity is observed for NS3. A sample is positive for $\mathrm{HCV}$ antibodies if at least two HCV antigen lines have reactivity ratings of \pm or higher. A sample is considered indeterminate for $\mathrm{HCV}$ antibodies if one HCV antigen line has a reactivity rating of $1+$ or higher, the NS3 line has a reactivity of \pm or higher and all other antigen lines are negative.

Other viral serological tests. At center A, for the LRG, the THG and the HCG, the HbsAg test was performed using a commercial kit (Surase B-96, General Biologicals Corp., Taiwan) by using a sandwich microelisa method. The HIV Ag\&Ab test was performed using a commercial kit (Genscreen ULTRA HIV Ag-Ab, Bio-Rad, France) in the TRITURUS system, which is automated open system (Diagnostics, Grifols, Spain) that uses the sandwich microelisa method. At center B, for the LRG, the THG and the HCG, the HbsAg test was performed using a commercial kit (Enzygnost HBsAg 6.0, Siemens Healthcare Diagnostics Products GmbH, Germany) with the sandwich microelisa method. The HIV Ag\&Ab test was performed using a commercial kit (Enzygnost HIV Integral II, Siemens Healthcare Diagnostics Products GmbH, Germany) in the TECAN Freedom Evolyzer 200 (Tecan, Switzerland), which is an automated open system (Diagnostics, Spain) that uses the sandwich microelisa method. Values above the cut-off value listed in the manufacturer's recommendations were considered to be reactive; values below the cut-off value were considered to be non-reactive.
HCV-RNA test. To detect HCV-RNA, a commercial kit (COBAS Ampliprep/COBAS TagMan HCV quantitative test, version 2.0, Roche, Switzerland) was used. The detection range of the kit was $15-108 \mathrm{IU} / \mathrm{ml}$.

Neopterin and sCD14 tests. The cut-off value of neopterin kit, which has a $0.79 \mathrm{nmol} / \mathrm{l}$ analytical sensitivity value, was according to the literature generally regarded as $10 \mathrm{nmol} / \mathrm{l}$ and levels above this cut-off value were accepted as pathological in related studies (Eisenhut, 2013). However, we did not use $10 \mathrm{nmol} / \mathrm{l}$ cut-off values for neopterin and a cut-off value of $5.36 \mathrm{nmol} / \mathrm{l}$ was calculated from receiver operating characteristic curves (ROC) for predicting HIV-1 infection. When levels were above this cut-off value, samples were considered as positive. Serum samples were analyzed using a commercial neopterin test kit (DRG Instruments GMBH, Germany) in the TRITURUS device using the quantitative competitive EIA method. Serum samples were analyzed for SCD14 using an Avizcera (Bioscience, USA) commercial kit in the TRITURUS device using the quantitative sandwich EIA method. A cut-off value of 3.95 $\mu \mathrm{g} / \mathrm{ml}$ for sCD14 was calculated from ROC for predicting HIV-1 infection. When levels were above this cut-off value, presence of infection was considered as positive.

Statistical methods. Non-parametric Kruskal-Wallis One-way ANOVA with a post-hoc Tukey HSD test for multiple comparisons was used to compare subgroups for means of quantitative variables. A chi square test was evaluated for categorical variables. Pearson correlation coefficients were calculated to characterize relationships between each quantitative variable within groups. The ROC were analyzed to determine diagnostic validity of the quantitative variables. All statistical analyses were calculated using IBM SPSS version 20 (IBM Corporation, USA) and MedCalc version 13 (MedCalc, Belgium) statistical software packages. Significance was recognized when $\mathrm{p}<0.05$. Performance parameters of the neopterin and SCD14 tests (sensitivity, specificity, and positive and negative predictive values) between the diagnoses of $\mathrm{HCV}$ infections were assessed using the kappa test. An agreement limit of at least $65 \%$ was used as the base value.

\section{Results}

Baseline characteristics of all groups are shown in Table 1. Neopterin and sCD14 levels among the THG, HCG, and those

Table 1. Baseline characteristics of study and control groups

\begin{tabular}{lccc}
\hline Characteristics & $\begin{array}{c}\text { Low anti-HCV reactivity group } \\
(\mathbf{n}: \mathbf{7 0})\end{array}$ & $\begin{array}{c}\text { True positive HCV } \\
\text { infection group } \\
(\mathbf{n}: \mathbf{3 0})\end{array}$ & $\begin{array}{c}\text { Healthy control group } \\
(\mathbf{n}: 30)\end{array}$ \\
\hline Age: Median (min-max) & $34.5(18-78)$ & $36.5(19-56)$ & $34(20-57)$ \\
Male (\%) & $47(67.14 \%)$ & $20(66.6 \%)$ & $19(63.33 \%)$ \\
Female (\%) & $23(32.8 \%)$ & $10(33.34 \%)$ & $11(36.67 \%)$ \\
AST: Median (min-max) & $19(12-51)$ & $58(17-245)$ & $18(10-39)$ \\
ALT: Median (min-max) & $17.5(7-47)$ & $59(23-383)$ & $19(9-38)$ \\
\hline
\end{tabular}

Data are given as $n$ (percent) or value (range). 
Table 2. Comparison of mean neopterin and sCD14 levels in study and control groups

\begin{tabular}{|c|c|c|}
\hline \multirow{2}{*}{ Groups } & \multicolumn{2}{|c|}{ Markers } \\
\hline & Neopterin & sCD14 \\
\hline LRG (70) & $5.06 \pm 1.07(3.15-10.18)$ & $6.95 \pm 1.46(2.93-9.74)$ \\
\hline THG (30) & $6.46 \pm 1.76(4.44-12.62)$ & $6.07 \pm 1.08(4.14-9.22)$ \\
\hline \multirow[t]{2}{*}{ HCG (30) } & $4.99 \pm 1.07(3.02-8.55)$ & $3.76 \pm 1.41(1.85-6.98)$ \\
\hline & \multicolumn{2}{|c|}{ Statistical value } \\
\hline LRGxTHG & $\mathrm{p}<0.001$ & $\mathrm{p}<0.05$ \\
\hline LRGxHCG & $\mathrm{p}>0.05$ & $\mathrm{p}<0.001$ \\
\hline THGxHCG & $\mathrm{p}<0.001$ & $\mathrm{p}<0.001$ \\
\hline
\end{tabular}

LRG: low anti-HCV reactivity group; THG: true positive HCV infection group, HCG: healthy control group, sd: standart deviation.

with the low anti-HCV reactivity group patterns are shown in Table 2. The mean $\pm \mathrm{sd}$ ( $\min -\mathrm{max})$ neopterin levels of the LRG, THG and HCG were determined to be 5.06 \pm 1.07 (3.15-10.18), $6.46 \pm 1.76(4.44-12.62)$ and $4.99 \pm 1.07$ (3.02-8.55), respectively. In the present study, we found that the mean neopterin levels of LRG patients were significantly lower than those of THG ( $\mathrm{p}<0.001)$ patients. In contrast, those values were not significantly different from those of the HCG $(p>0.05)$. The mean \pm s.d. (min-max) sCD14 levels of the LRG, THG and HCG were determined to be $6.95 \pm 1.46$ (2.93-9.74), $6.07 \pm$ 1.08 (4.14-9.22) and $3.76 \pm 1.41$ (1.85-6.98), respectively. The mean sCD14 level was significantly higher in the LRG than in the THG and the HCG $(\mathrm{p}<0.05, \mathrm{p}<0.001)$ (Table 2, Fig. 1).

A cut-off value of neopterin and sCD14 was calculated from ROC for predicting true positive $\mathrm{HCV}$ infection. When levels were above this cut-off value, presence of true $\mathrm{HCV}$ infection was considered. Various sCD14 and neopterin cutoff values were examined or prediction of HCV infection, and values of $3.95 \mu \mathrm{g} / \mathrm{ml}$ and $5.36 \mathrm{nmol} / \mathrm{l}$ resulted in the maximum area under the ROC, which were 0.859 (95\% CI, 0.745 to 0.935 ; <0.0001) and 0.788 (95\% CI, 0.663 to 0.883 ; $<0.0001$ ), respectively (Fig. 1). These cut-offs corresponded to a sensitivity of $73.3 \%$ and a specificity of $73.3 \%$ for neopterin and of $100 \%$ and $76.7 \%$ for sCD14.

Neopterin had an adequate predictive value for identifying true positive HCV infections among the THG than suspected

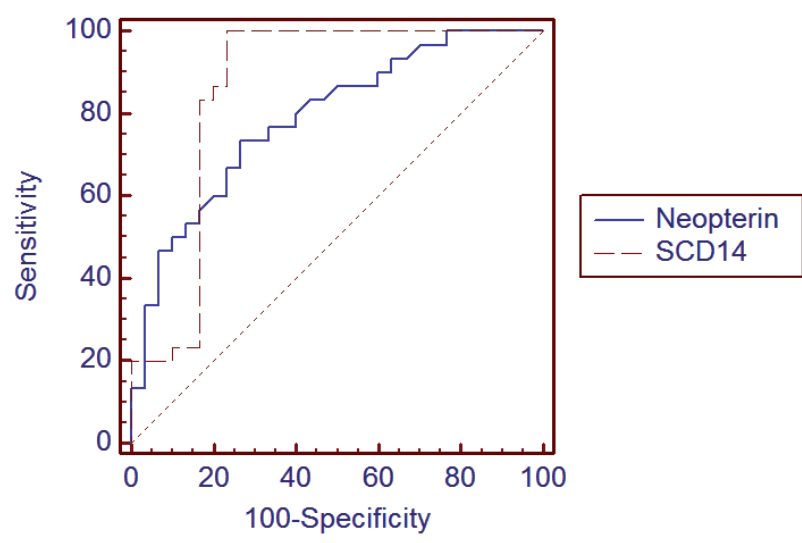

Fig. 1

Receiver operating characteristic curves showing areas under the curve for neopterin and sCD14 for predicting $\mathrm{HCV}$ infection The neopterin cut-off value is $5.36 \mathrm{nmol} / \mathrm{l}$, and the $\mathrm{SCD} 14$ cut-off value is $3.95 \mu \mathrm{g} / \mathrm{ml}$. LRG: low anti-HCV reactivity group; THG; true positive HCV infection group, HCG; healthy control group.

low anti-HCV reactivity group. Mean sCD14 were significantly higher in LRG than THG and HCG. Therefore sCD14 seems not to be a useful marker for identifying true positive $\mathrm{HCV}$ infections. When the diagnostic performance of both neopterin and sCD14 as surrogate tests for HCV diagnosis were examined, the sensitivity, specificity, positive (PPV) and negative predictive values (NPV) were determined. For neopterin, they were determined to be $73.3 \%, 73.3 \%$, $73.3 \%$, and $73.3 \%$, respectively. The corresponding values for sCD14 were determined to be $100 \%, 76.7 \%, 81.1 \%$, $83.3 \%$, and $76.7 \%$, respectively (Table 3 ).

\section{Discussion}

Neopterin and sCD14 levels may indicate the activation status of an individual's cellular immune system. The cellular immune system plays an important role in the pathogenesis and progression of various infectious diseases. Neopterin and sCD14 detection for non-specific conditions such as inflam-

Table 3. Diagnostic performances of neopterin and sCD14 in true positive HCV infection group

\begin{tabular}{lccccccc}
\hline & Cut-off value & AUC (95\%CI) & Se. (\%) & Spe. (\%) & PPV & NPV & p value \\
\hline Neopterin, nmol/1 & 5.36 & $\begin{array}{c}0.788 \\
(0.663-0.833)\end{array}$ & $73.3 \%$ & $73.3 \%$ & $73.3 \%$ & $73.3 \%$ & $<0.0001$ \\
\hline sCD14, g/ml & 3.95 & $\begin{array}{c}0.859 \\
(0.745-0.935)\end{array}$ & $100 \%$ & $76.7 \%$ & $81.1 \%$ & $76.7 \%$ & $<0.0001$ \\
\hline
\end{tabular}

Se.: sensitivity; Spe.: specificity; AUC: area underthe curve; PPV: positive predictive value; NPV: negative predictive value; CI: confidence interval. 
mation (which is indicative of several different diseases) may be useful to screen blood used in blood banks.

In recent years, there has been a remarkable increase in the number of researchers who have attempted to develop serological, molecular, and surrogate tests for the diagnosis of HCV infections. Surrogate tests provide researchers with reasonable testing options; these tests are suitable and effective for a number of reasons, including easy access, less expense, and rapid test results. In recent years, the focus has been on neopterin, which appears to be useful for the early diagnostics of viral infections (such as cytomeglovirus and parvovirus 19) and in donor scans for blood-borne infections at blood banks; more recently, it has been suggested that sCD14 can have the same use as neopterin (Murr et al., 2002; Bas et al., 2004).

We tried to understand the difference between a true positive $\mathrm{HCV}$ infection and low anti-HCV reactivity by using neopterin and sCD14 levels. ELISA kits have high rates of false positive results, especially in populations with a low HCV prevalence. RIBA results in combination with low-reactive anti-HCV results have also been linked to false positive results in low HCV risk individuals (Kesli et al., 2009). One suggestion for solving this problem is to use HCV RNA as a primary test (Lok et al., 1997; Dufour et al., 2000). In 2003, the CDC (Alter et al., 2003) recommended the use of a signal-to-cut-off ratio (s/co) of 3.8 to provide $\mathrm{a} \geq 95 \%$ positive predictive value and defined reactivities $<3.8$ as low-reactive anti-HCV results. In addition, it was reported that the majority of low-reactivity results could represent false positive results; this type of result accounted for a serious problem in HCV diagnostics. Previous viral infections, autoimmune diseases, allergies, influenza vaccines, and immunoglobulin treatments have been proposed as reasons for such low-reactive false-positive results (Mullis et al., 2013; Takeshita et al., 2000; Egerer et al., 2000). Both EIA and RIBA may give indeterminate results for HCV diagnosis. In order to understand mechanisms underlying HCV pathogenesis, we have to evaluate basic immunological mechanisms that are active during an HCV infection. From the point of view of innate immunity, NK cells are usually activated in an early phase of acute $\mathrm{HCV}$ infection and also in a chronic $\mathrm{HCV}$ infection. NK cells are abundant in the liver, and activated NK cells recruit virus specific T cells and induce antiviral immunity in the liver. NK cells destroy virusinfected hepatocytes directly via cytolytic mechanisms and indirectly by secreting cytokines including IFN- $\gamma$ and TNF-a (Irshad et al., 2013). HCV fragments, which are $\mathrm{HCV}$-infected hepatocytes that have been released and destroyed, are taken up by myeloid DCs. These DCs migrate to draining lymph nodes where they express HCV antigens on HLA class II molecules. After that, they stimulate the expression of co-stimulatory molecules (CD80 and CD86) which interact with and activate antigen-specific Th cells (Malta et al., 2013).

In HCV infections, activated Th cells induce DC maturation. Mature DCs induce T-cell activation by causing overexpression of their surface molecules. DCs also increase antigen presentation as a result of cytokine production that stimulates T-cell activation. IL-12 has been shown to play an important role in stimulating IFN- $\gamma$ production from activated T cells (Jaime-Ramirez et al., 2011; Heufler et al., 1996). Also, in acute HCV infections, effective virus clearance depends on a strong Th1 immune response. In contrast, people who lack IL-12 and IFN- $\gamma$ production appear to develop chronic viral persistence (Testino et al., 2013; Valiante et al., 2000). Neopterin is released from monocytes, macrophages, DCs, and endothelial cells, all of which are activated by IFN- $\gamma$ secreted by Th1 lymphocytes (Hoffmann et al., 2004; Schroecksnadel et al., 2004). Increased neopterin production might be induced by an HCV infection, but there may be other mechanisms which also produce neopterin increase during HCV infections. In this study, we selected a chronic HCV infection group.

In exploring other mechanisms, especially in chronic $\mathrm{HCV}$ infections, it has been suggested that the gastrointestinal system might be a reservoir for HCV and a possible source of re-infection for hepatocytes through portal vein blood (Miglioresi et al., 2003). Translocation of microbial products into the peripheral circulation may cause the loss of gut-associated lymphoid tissue integrity. Translocation of microbial products via LPS-induced monocyte activation may result in chronic immune activation during both $\mathrm{HIV}$ and HCV infections. An increase in neopterin levels may accompany this process. On the other hand, different mechanisms during HCV infections that can cause neopterin increase have also been proposed. These mechanisms include abundant proinflammatory cytokine production resulting in gastrointestinal inflammation, dysregulation of $\mathrm{T}$ cell subsets, and decreased microbial clearance (Shata et al., 2013).

On the other hand, the increased expression of NKp30 on NK cells suggests a level of activation of NK cells in chronically viremic HCV monoinfected subjects. This mechanism may suggest the increased neopterin levels in chronically HCV infected patients (Kottilil et al., 2009). Immune activation may also be induced by an increase in regulatory $\mathrm{T}$ lymphocytes (Treg) (Aandahl et al., 2004). Cell contact and Treg-dependent secretion of transforming growth factor beta 1 (TGF- $\beta 1$ ) modulate the lymphocyte proliferation which may contribute to the immune response. HCV might use Treg-dependent TGF- $\beta 1$ secretion as a way to produce an increase in neopterin levels (Shevach, 2006). In a study by Márquez et al. (2015) patients with a chronic HIV infection showed a persistent increase in intestinal permeability and immune modifications. HCV-associated cirrhosis was asso- 
ciated with more elevated serum levels of monocyte-derived markers (sCD14 and IL-6). Furthermore, cirrhosis influenced these patients' continued immune reconstitution.

There are a limited number of studies concerning HCV infections and neopterin levels. In a previous study, ZwirskaKorczala et al. (2005) reported that serum neopterin levels of patients with chronic HCV infection were significantly higher than that in HCG patients $(\mathrm{p}<0.01)$. Schennach et al. (1998) determined neopterin to be positive in 44 of 328 cases of positive anti-HCV test results, eight of 39 RIBA-positive cases, and seven of 19 PCR-positive cases. These authors suggested that there could be a significant relationship between anti-HCV positivity and positive PCR results. Prior et al. (1987) studied urinary neopterin levels in non-A and -B hepatitis patients and those with non-infectious fatty liver syndrome. High neopterin levels in $23(88.5 \%)$ of 26 non-A and -B hepatitis patients and only one (6\%) in 16 fatty liver syndrome patients were detected. In a similar study, serum neopterin levels of HCV patients were significantly higher than those of HCV-negative patients $(\mathrm{p}<0.05)$ (Weiss et al., 1999). Althought ROC curve analysis for predicting $\mathrm{HCV}$ infection using neopterin was statistically significant (AUC: $0.788 ; \mathrm{p}<0.0001$ ); these results, however, were not promising for using neopterin as a surrogate test. The sensitivity and specificity of neopterin was average (73\%) when $5.36 \mathrm{nmol} / \mathrm{l}$ was chosen as the cut-off value. We suggest that neopterin is not useful as a diagnostic marker, but its moderate sensitivity and specificity may be useful for deciding whether a case is a true positive HCV infection. We determined that mean neopterin levels of individuals with low anti-HCV reactivity were significantly lower than those of the patients with true positive HCV infections ( $\mathrm{p}<0.001)$. HCV-DNA negativity in all LRG cases supports that LRG with different RIBA patterns indicates that they are not true HCV infections.

In chronic HCV infections, several reports support the idea that microbial translocation may be the reason for elevated sCD14 levels. IL-6, a marker of hepatic inflammation, decreased with $\mathrm{HCV}$ infection treatment, and high plasma sCD14 levels predicted disease progression in $\mathrm{HCV}$ infection. LPS bioreactivity, as indicated by sCD14 levels, distinguished patients with severe liver fibrosis from $\mathrm{HCV}$ patients and correlated with markers of hepatic inflammation and fibrosis. Also, microbial translocation, LPS-induced monocyte activation, and IL-6 levels were increased in HCV infections (Sandler et al., 2011). sCD14 was previously thought to originate from monocytes/macrophages. Studies in mice and rats, however, suggested that CD14 can also be expressed by hepatocytes. In rat and mouse models, hepatocytes were indicated as a possible source of sCD14. However, there is no direct evidence showing that human hepatocytes also express CD14 in vivo and release sCD14 into the plasma. On the other hand, in vitro studies of hu- man hepatocytes showed that these cells have the capability to produce sCD14. Elevated SCD14 concentrations have been detected in plasma from patients with chronic HCV (Jirillo et al., 1998; Meuleman et al., 2006; Su et al., 1999; Hetherington et al., 1999).

Meuleman et al. (2006) showed for the first time that human hepatocytes in vivo were able to secrete sCD14 into the circulation. They also suggested that a large fraction of the sCD14 in human plasma originates from the liver. It is plausible that the liver also produces a series of acute-phase markers. In a study related to sCD14 levels, Steyaert et al. (2003) reported that there was a significant difference between patients with chronic HCV and those with elevated HCG levels ( $\mathrm{p}<0.001$ ). In another study with chronic HCV patients, the mean sCD14 level of $13 \mathrm{HCV}$ patients was slightly higher than that of 52 HCG patients $(\mathrm{p}=0.01)$ (Oesterreicher et al., 1995). ROC analysis of sCD14 for the prediction of true HCV infection was significant ( $p<0.0001)$, and sCD14 also had high sensitivity (100\%) but moderate specificity $(76.7 \%)$ for identifying those with true $\mathrm{HCV}$ (AUC $=0.859)$ infection. While the sensitivity of sCD14 was very high (100\%), its specificity was moderate $(76.7 \%)$ when $3.95 \mathrm{~g} / \mathrm{ml}$ was chosen as the cut-off value. We suggest that sCD14 may not be useful as a diagnostic marker. We suggest that sCD14 may not be useful as a non-specific marker for indirect assessment of immune activation status among patients whether or not they have true positive HCV infections. Because of the mean sCD14 level was significantly higher in LRG than in THG and the HCG ( $<<0.05, \mathrm{p}<0.001)$ in this study. The existence of higher neopterin and sCD14 levels in the true positive HCV group rather than HCG is consistent with other reports in the literature. However, the presence of similar neopterin levels in the low anti-HCV reactivity group and the HCG suggested that there was no antigenic stimulation related to $\mathrm{HCV}$; therefore, this finding suggests that there was no macrophage activation connected with Th1 on the basis of TNF- $a$. Hence, the 100\% HCV-DNA negativity in this group supports this condition. However, in the true positive HCV group, the presence of high neopterin levels suggested that the Th1-macrophage pathway was active and linked to HCV induction. In the true HCV infection group, high sCD14 levels could be a consequence of human hepatocytes' expression of CD14 in vivo and release of sCD14 into the plasma. As an alternative mechanism, translocation of microbial products via LPS-induced monocyte activation may result in chronic immune activation during $\mathrm{HCV}$ as well as HIV infections.

The detection of significantly higher SCD14 levels in LRG and THG patients rather than in HCG patients has brought into question the use of paradoxical evaluations in these cases. The detection of negative HCV-RNA test results in the LRG demonstrated that these patients had no real HCV infections. In contrast, sCD14 levels were found to be higher 
in THG patients with real HCV infections. This condition may be related to an unknown factor or to some underlying disease (Kawasaki disease, atopic dermatitis, liver diseases, rheumatoid arthritis, or systemic lupus erythematous).

Thus, the detection of low-reactive (false positivity) anti-HCV results in LRG patients is thought to support nonspecific exposure. We propose that some underlying factors specific to these cases could have non-specifically affected the anti-HCV results and sCD14 levels. In addition, based on the idea that statistical significance might not reflect clinical significance, we suggest that significantly higher sCD14 levels that were detected in LRG patients might not indicate the presence of a real HCV infection.

In the literature, we could not find any study related to diagnostic performance parameters. Only Schennach et al. (1998) from Austria reported a study of 54,402 blood donors in which it was shown that the possibility of the HCV-RNA test being positive was seven times greater for patients with neopterin levels over $10 \mathrm{nmol} / \mathrm{l}$ than for patients whose value was below $10 \mathrm{nmol} / \mathrm{l}$. We suggest that the neopterin test cannot be beneficial as a surrogate test in patients with true $\mathrm{HCV}$ infections and anti-HCV low-reactive/different RIBA patterns. Nonetheless, we suggest that it could be used as an additional test for anti-HCV in blood donor screenings. In our study, neopterin levels did not suggest a specific HCV infection period based on immune activation in patients with low anti-HCV reactivity; additional studies however, which include a large series of cases with matched patients, are required. Nonetheless, the false-positive results of the sCD14 test in LRG patients and the detection of sCD14 at high levels in chronic HCV patients suggest that this test was probably affected in a non-specific manner, particularly in some underlying immunohematological atypical pathologies.

Despite their high analytical and diagnostic sensitivity, the application of nucleic acid tests for blood safety at blood banks remains controversial due to its high cost and long application time. These findings suggest that only neopterin may be used to exclude low reactivity anti-HCV results with different RIBA patterns from true HCV infection possibilities. We suggest that neopterin may be useful as an indirect, non-specific marker for predicting an individual's immune activation status whether or not the individual has a true positive HCV infection. Therefore, the results of this study suggest that the use of neopterin, rather than sCD14, in combination with fourth-generation EIA/CMIA combination tests will be useful in situations where nucleic acid tests are not available for the screening of blood donors at blood banks.

Acknowledgement. This work was supported by Istanbul University Research Fund with the project No. 45694. This research was presented as a poster in the 17th International Congress On Infectious Diseases (ICID) Hayderabad, 2016. We also thank Prof.
Dr Suphi Vehid (Cerrahpasa Medical Faculty, Turkey) and Esad Bonabi (Aydin University, Turkey) for their technical assistance related to the poster presentation in the 17th ICID.

\section{References}

Aandahl EM, Michaëlsson J, Moretto WJ, Hecht FM, Nixon DF (2004): Human CD4+ CD25+ regulatory T cells control T-cell responses to human immunodeficiency virus and cytomegalovirus antigens. J. Virol. 78, 2454-2459. https:// doi.org/10.1128/JVI.78.5.2454-2459.2004

Alter MJ, Kuhnert WL, Finelli L (2003): Guidelines for laboratory testing and result reporting of antibody to hepatitis $\mathrm{C}$ virus. Centers for Disease Control and Prevention. MMWR Recomm. Rep. 52, 1-13.

Bas S, Gauthier BR, Spenato U, Stingelin S, Gabay C (2004): CD14 is an acute-phase protein. J. Immunol. 172, 4470-4479. https://doi.org/10.4049/jimmunol.172.7.4470

Caruntu FA, Benea L. (2006): Acute hepatitis C virus infection: Diagnosis, pathogenesis, treatment. J. Gastrointestin. Liver. Dis. 15, 249-256.

Dufour DR, Lott JA, Nolte FS, Gretch DR, Koff RS, Seeff LB (2000): Diagnosis and monitoring of hepatic injury: I. Characteristics of laboratory tests. Clin. Chem. 46, 2027-2049.

Egerer K, Feist E, Rohr U, Pruss A, Burmester GR, Dörner T (2000): Increased serum soluble CD14, ICAM-1 and E-selectin correlate with disease activity and prognosis in systemic lupus erythematosus. Lupus 9, 614-621. https://doi. org/10.1191/096120300678828749

Eisenhut M (2013): Neopterin in diagnosis and monitoring of infectious diseases. J. Biomark. 2013, 96432. https://doi. org $/ 10.1155 / 2013 / 196432$

Fuchs D, Weiss G, Reibneggar G, Wachter H (1992): The role of neopterin as a monitor of cellular immune activation in transplantation, inflammatory, infectious, and malignant diseases. Crit. Rev. Clin. Lab. Sci. 29, 307-341. https://doi. org/10.3109/10408369209114604

Hetherington CJ, Kingsley PD, Crocicchio F, Zhang P, Rabin MS Palis J, Zhang DE (1999): Characterization of human endotoxin lipopolysaccharide receptor CD14 expression in transgenic mice. J. Immunol. 162, 503-509.

Heufler C, Koch F, Stanzl U, Topar G, Wysocka M, Trinchieri G, Enk A, Steinman RM, Romani N, Schuler G (1996): Interleukin-12 is produced by dendritic cells and mediates Thelper 1 development as well as interferon-gamma productionby T helper 1 cells. Eur. J. Immunol. 26, 659-668. https://doi.org/10.1002/eji.1830260323

Hoffmann G, Wirleitner B, Fuchs D (2003): Potential role of immune system activation- associated production of neopterin derivatives in humans. Inflamm. Res. 52, 313-321. https://doi.org/10.1007/s00011-003-1181-9

Hoffmann G, Schobersberger W (2004): Neopterin: A mediator of the cellular immune system. Pteridines 15, 107-112. https://doi.org/10.1515/pteridines.2004.15.3.107

Irshad M, Mankotia DS, Irshad K (2013): An insight into the diagnosis and pathogenesis of hepatitis $\mathrm{C}$ virus infec- 
tion. World. J. Gastroenterol. 19, 7896-7909. https://doi. org/10.3748/wjg.v19.i44.7896

Jaime-Ramirez AC, Mundy-Bosse BL, Kondadasula S, Jones NB, Roda JM, Mani A, Parihar R, Karpa V, Papenfuss TL, LaPerle KM, Biller E, Lehman A, Chaudhury AR, Jarjoura D, Burry RW, Carson WE (2011): IL-12 enhances the antitumor actions of trastuzumab via NK cell IFN- $\gamma$ production. J. Immunol. 186, 3401-3409. https://doi. org/10.4049/jimmunol.1000328

Jirillo E, Amati L, Caradonna L, Greco B, Cozzolongo R, Cuppone R, Piazzolla G, Caccavo D, Antonaci S, Manghisi OG (1998): Soluble (s) CD14 and plasmatic lipopolysaccharides (LPS) in patients with chronic hepatitis C before and after treatment with interferon (IFN)-alpha. Immunopharmacol. Immunotoxicol. 20, 1-14. https:// doi.org/10.3109/08923979809034805

Kesli R, Ozdemir M, Kurtoglu MG, Baykan M, Baysal B (2009): Evaluation and comparison of three different antihepatitis $\mathrm{C}$ virus antibody tests based on chemiluminescence and enzyme-linked immunosorbent assay methods used in the diagnosis of hepatitis $\mathrm{C}$ infections in Turkey. J. Int. Med. Res. 37, 1420-1429. https://doi. org/10.1177/147323000903700516

Kottilil S, Yan MY, Reitano KN, Zhang X, Lempicki R, Roby G, Daucher M, Yang J, Cortez KJ, Ghany M, Polis MA, Fauci AS (2009): Human immunodeficiency virus and hepatitis $\mathrm{C}$ infections induce distinct immunologic imprints in peripheral mononuclear cells. Hepatology 50, 34-45. https://doi.org/10.1002/hep.23055

Lien E, Aukrust P, Sundan A, Müller F, Frøland SS, Espevik T (1998): Elevated levels of serum-soluble CD14 in human immunodeficiency virus type 1 (HIV-1) infection: correlation to disease progression and clinical events. Blood. 92, 2084-2092.

Lok AS, Gunaratnam NT (1997): Diagnosis of hepatitis C. Hepatology. 26, 48S-56S. https://doi.org/10.1002/ hep. 510260709

Malta FM, Bruno FR, Carvalho KI, Nastri AC, Kalil J, Carrilho FJ, Kallas EG, Pinho JR (2013): HCV viremia drives an increment of CD86 expression by myeloid dendritic cells. J. Med. Virol. 85, 1919-1924. https://doi.org/10.1002/jmv.23692

Márquez M, Romero-Cores $\mathrm{P}$, Montes-Oca M, Martín-Aspas A, Soto-Cárdenas MJ, Guerrero F, Fernández-Gutiérrez C, Girón-González JA (2015): Immune activation response in chronic HIV-infected patients: influence of Hepatitis C virus coinfection. PLoS. One 10, e0119568. https://doi. org/10.1371/journal.pone.0119568

Medhat E, Salama H, Fouad H, Abd El Haleem H, Said M, ElNahaas SM, Omran D (2015): Serum soluble CD14 in Egyptian patients with chronic hepatitis C: its relationship to disease progression and response to treatment. J. Interferon. Cytokine. Res. 35, 563-568. https://doi. org/10.1089/jir.2014.0155

Meuleman P, Steyaert S, Libbrecht L, Couvent S, Van Houtte F, Clinckspoor F, de Hemptinne B, Roskams T, Vanlandschoot P, Leroux-Roels G (2006): Human hepatocytes secrete soluble CD14, a process not directly influenced by HBV and HCV infection. Clin. Chim. Acta 366, 156-162. https://doi.org/10.1016/j.cca.2005.09.022
Miglioresi L, Riva E, Antonelli G, Russo F, Ricci GL (2003): Localization of hepatitis $\mathrm{C}$ virus in gastrointestinal mucosa: a possible reservoir for relapse. Hepatology 38,775 . https://doi.org/10.1053/jhep.2003.50322

Moyer LA, Mast EE, Alter MJ (1999): Hepatitis C: Part I. Routine serologic testing and diagnosis. Am. Fam. Physician. 59, 79-88.

Mullis CE, Laeyendecker O, Reynolds SJ, Ocama P, Quinn J, Boaz I, Gray RH, Kirk GD, Thomas DL, Quinn TC, Stabinski L (2013): High frequency of false-positive hepatitis C virus enzyme-linked immunosorbent assay in Rakai, Uganda. Clin. Infect. Dis. 57, 1747-1750. https://doi.org/10.1093/ cid/cit602

Murr C, Widner B, Wirleitner B, Fuchs D (2002): Neopterin as a marker for immune system activation. Curr. Drug. Metab. 3, 175-187. https://doi.org/10.2174/1389200024605082

Oesterreicher C, Pfeffel F, Petermann D, Müller C (1995): Increased in vitro production and serum levels of the soluble LPS receptor sCD14 in liver diseases. J. Hepatol. 23, 396-402. https://doi.org/10.1016/0168-8278(95)80197-9

Oethinger M, Mayo DR, Falcone JA, Barua PK, Griffith BP (2005): Efficiency of the ortho VITROS assay for detection of hepatitis $\mathrm{C}$ virus-specific antibodies increased by elimination of supplemental testing of samples with very low sample-to-cutoff ratios. J. Clin. Microbiol. 43, 2477-2480. https://doi.org/10.1128/jcm.43.5.2477-2480.2005

Pawlotsky JM, Lonjon I, Hezode C, Raynard B, Darthuy F, Remire J, Soussy CJ, Dhumeaux D (1998): What strategy should be used for diagnosis of hepatitis $\mathrm{C}$ virus infection in clinical laboratories? Hepatology 27, 1700-1702. https:// doi.org/10.1002/hep. 510270632

Prior C, Fuchs D, Hausen A, Judmaier G, Reibnegger G, Werner ER, Vogel W, Wachter H (1987): Potential of urinary neopterin excretion in differentiating chronic non-A, non-B hepatitis from fatty liver. Lancet 2, 1235-1237. https://doi. org/10.1016/S0140-6736(87)91852-6

Sandler NG, Koh C, Roque A, Eccleston JL, Siegel RB, Demino M, Kleiner DE, Deeks SG, Liang TJ, Heller T, Douek DC (2011). Host response to translocated microbial products predicts outcomes of patients with $\mathrm{HBV}$ or $\mathrm{HCV}$ infection. Gastroenterology 141, 1220-1230. https://doi. org/10.1053/j.gastro.2011.06.063

Schennach H, Schoenitzer D, Fuchs D (1998). Association between chronic hepatitis $\mathrm{C}$ virus infection and increased neopterin concentrations in blood donations. Clin. Chem. 44, 2225-2226.

Schroecksnadel K, Murr C, Winkler C, Wirleitner B, Fuith LC, Fuchs D (2004): Neopterin to monitor clinical pathologies involving interferon $\gamma$ production. Pteridines 15, 75-90. https://doi.org/10.1515/pteridines.2004.15.3.75

Shata MT, Abdel-Hameed EA, Hetta HF, Sherman KE (2013): Immune activation in HIV/HCV-infected patients is associated with low-level expression of liver expressed antimicrobial peptide-2 (LEAP-2). J. Clin. Pathol. 66, 967-975. https://doi.org/10.1136/jclinpath-2013-201581

Shevach EM (2006): From vanilla to 28 flavors: multiple varieties of T regulatory cells. Immunity 25, 195-201. https://doi. org/10.1016/j.immuni.2006.08.003 
Steyaert S, Vanlandschoot P, Van Vlierberghe H, Diepolder H, Leroux-Roels G (2003): Soluble CD14 levels are increased and inversely correlated with the levels of hepatitis B surface antigen in chronic hepatitis B patients. J. Med. Virol. 71, 188-194. https://doi.org/10.1002/jmv.10469

Su GL, Dorko K, Strom SC, Nussler AK, Wang SC (1999): CD14 expression and production by human hepatocytes. J. Hepatol. 31, 435-442. Takeshita S, Nakatani K, Tsujimoto H, Kawamura Y, Kawase H, Sekine I (2000): Increased levels of circulating soluble CD14 in Kawasaki disease. Clin. Exp. Immunol. 119, 376-381.

Testino G, Sumberaz A, Leone S, Borro P (2013): Recurrent hepatitis $\mathrm{C}$ and non-alcoholic fatty liver disease in transplanted patients: a review. Minerva. Med. 104, 225-232.
Valiante NM, D'Andrea A, Crotta S, Lechner F, Klenerman P, Nuti S, Wack A, Abrignani S (2000): Life, activation and death of intrahepatic lymphocytes in chronic hepatitis C. Immunol. Rev. 174, 77-89. https://doi.org/10.1034/j.1600$\underline{0528.2002 .017417 . \mathrm{x}}$

Weiss G, Umlauft F, Urbanek M, Herold M, Loyevsky M, Offner F, Gordeuk VR (1999): Associations between cellular immune effector function, iron metabolism, and disease activity in patients with chronic hepatitis $\mathrm{C}$ virus infection. J. Infect. Dis. 180, 1452-1458. https://doi.org/10.1086/315052

Zwirska-Korczala K, Kukla M, Ziołkowski A (2005): Leptin, neopterin and hepatocyte growth factor as markers of fibrosis and inflammatory activity in chronic hepatitis C. Exp. Clin. Hep. 1, 60-65. 